\title{
Appel urgent à l'action pour limiter la hausse de la température mondiale, restaurer la biodiversité et protéger la santé : les pays riches doivent en faire beaucoup plus, et plus rapidement
}

\author{
Lukoye Atwoli ${ }^{1}$, Abdullah H. Baqui ${ }^{2}$, Thomas Benfield ${ }^{3}$, Raffaella Bosurgi ${ }^{4}$, Fiona Godlee ${ }^{5}$, \\ Stephen Hancocks ${ }^{6}$, Richard Horton ${ }^{7}$, Laurie Laybourn-Langton ${ }^{8 *}$, Carlos Augusto Monteiro 9 , \\ Ian Norman ${ }^{10}$, Kirsten Patrick ${ }^{11}$, Nigel Praities ${ }^{12}$, Marcel GM Olde Rikkert ${ }^{13}$, Eric J Rubin ${ }^{14}$, \\ Peush Sahni ${ }^{15}$, Richard Smith ${ }^{16}$, Nicholas J. Talley ${ }^{17}$, Sue Turale ${ }^{18}$, Damián Vázquez ${ }^{19}$
}

*Correspondance : laurie.laybourn@ukhealthalliance.org

Citation proposée : Atwoli L, Baqui AH, Benfield T, Bosurgi R, Godlee F, Hancocks S, Horton R, Laybourn-Langton L, Monteiro CA, Normal I, Patrick K, Praities N, Rikkert MGMO, Rubin EJ, Sahni P, Smith R, Talley NJ, Turale S, Vázquez D. Appel urgent à l'action pour limiter la hausse de la température mondiale, restaurer la biodiversité et protéger la santé : les pays riches doivent en faire beaucoup plus, et plus rapidement. Relevé des maladies transmissibles au Canada 2021;47(11):492-5. https://doi.org/10.14745/ccdr.v47i11a01f

Mots-clés : mondial, changement climatique, biodiversité, santé, changements environnementaux

L'Assemblée générale des Nations Unies de septembre 2021 réunira les pays à un moment décisif pour une mobilisation collective afin de s'attaquer à la crise climatique planétaire. Ils se réuniront à nouveau pour le sommet sur la biodiversité à Kunming, en Chine, puis pour la Conférence sur les changements climatiques (COP26) à Glasgow, au Royaume-Uni. En prévision de ces rencontres majeures, nous, les rédacteurs en chef de revues médicales de partout sur la planète, lançons un appel urgent à l'action pour maintenir la hausse de la température mondiale moyenne en deçà de $1,5^{\circ} \mathrm{C}$, mettre un terme à la destruction des milieux naturels et protéger la santé.

Déjà, la santé est affectée par les hausses de la température mondiale et la destruction des écosystèmes, une situation que les professionnels de la santé dénoncent depuis des décennies (1). La science est sans équivoque : une augmentation mondiale de $1,5^{\circ} \mathrm{C}$ par rapport à la moyenne préindustrielle et les menaces constantes à la biodiversité représentent pour la santé un risque catastrophique qu'il sera impossible de renverser (2,3). Malgré toute l'attention que mérite encore la COVID-19, nous ne pouvons pas attendre la fin de la pandémie pour rapidement réduire les émissions.

Témoignant de la gravité du moment, cet éditorial est publié simultanément dans les grandes revues médicales du monde. Ensemble, nous reconnaissons que ce n'est qu'en apportant des changements fondamentaux et équitables à nos sociétés que nous pourrons renverser la trajectoire actuelle.

Les risques pour la santé d'une hausse de plus de $1,5^{\circ} \mathrm{C}$ de la température sont maintenant bien connus (2). En fait, aucune hausse de la température n'est « sécuritaire ». Au cours des 20 dernières années, la mortalité liée aux vagues de chaleur a augmenté d'au-delà de $50 \%$ chez les personnes de plus de 65 ans (4). La hausse des températures a causé une augmentation des problèmes de déshydratation et d'insuffisance rénale, des cancers de la peau, des infections tropicales, des problèmes de santé mentale, des complications de grossesse, des allergies et de la morbidité/ mortalité cardiovasculaires et pulmonaires $(5,6)$. Ces enjeux affectent de manière disproportionnée les plus vulnérables, soit les enfants, les personnes âgées, les minorités ethniques, les populations plus pauvres et les personnes ayant de problèmes de santé sous-jacents $(2,4)$.
Cette oeuvre est mise à la disposition selon les termes de la licence internationale Creative Commons Attribution 4.0

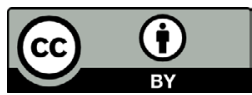

Rôles et affiliations

${ }^{1}$ Rédacteur en chef, East African Medical Journal

2 Rédacteur en chef, Journal of Health, Population and Nutrition

${ }^{3}$ Rédacteur en chef, Danish Medical Journal

${ }^{4}$ Rédactrice en chef, PLOS Medicine

${ }^{5}$ Rédactrice en chef, The BMJ

${ }^{6}$ Rédacteur en chef, British Dental Journal

${ }^{7}$ Rédacteur en chef, The Lancet

${ }^{8}$ Conseiller principal, UK Health Alliance on Climate Change

${ }^{9}$ Rédacteur en chef, Revista de Saúde Pública

${ }^{10}$ Rédacteur en chef, International Journal of Nursing Studies

${ }^{11}$ Rédactrice en chef par intérim, CMAJ

${ }^{12}$ Directeur général,

Pharmaceutical Journal

${ }^{13}$ Rédacteur en chef, Dutch Journal of Medicine

${ }^{14}$ Rédacteur en chef, NEJM

${ }^{15}$ Rédacteur en chef, National Medical Journal of India

${ }^{16}$ Président, UK Health Alliance on Climate Change

${ }^{17}$ Rédacteur en chef, Medical Journal of Australia

${ }^{18}$ Rédactrice en chef, International Nursing Review

${ }^{19}$ Rédacteur en chef, Pan American Journal of Public Health 
Le réchauffement climatique contribue aussi au déclin du potentiel de rendement mondial des principales cultures vivrières, qui ont diminué de 1,8 \%-5,6 \% depuis 1981; cette situation, combinée aux effets des phénomènes météorologiques extrêmes et à l'épuisement des sols, freine les efforts déployés pour combattre la malnutrition (4). La santé humaine est indissociable de celle des écosystèmes et la destruction galopante des milieux naturels, des habitats et des espèces, met en péril les réserves d'eau et d'aliments et exacerbe les risques de pandémies $(3,7,8)$.

Les conséquences de la crise environnementale touchent de manière disproportionnée les pays et les collectivités qui y ont le moins contribué et qui sont les moins en mesure d'y remédier. Or, aucun pays, peu importe sa richesse, ne sera à l'abri de ces impacts. Abandonner les plus vulnérables à ces conséquences disproportionnées ne fera qu'alimenter les conflits, aggraver l'insécurité alimentaire, provoquer des exodes forcés et laisser libre cours aux zoonoses; tout cela aura de graves répercussions sur tous les pays et toutes les communautés. Comme l'a révélé la pandémie de COVID-19, nous sommes globalement aussi forts que notre maillon le plus faible.

Toute hausse de plus de $1,5{ }^{\circ} \mathrm{C}$ nous rapproche d'un point de bascule pour les écosystèmes qui pourrait précipiter le monde dans une instabilité critique et nuirait grandement à notre capacité d'atténuer les effets délétères et d'éviter des cataclysmes environnementaux incontrôlables $(9,10)$.

\section{Les cibles mondiales sont insuffisantes}

Il est encourageant de constater que bon nombre de gouvernements, d'institutions financières et d'entreprises fixent des cibles de carboneutralité, notamment pour 2030, que le coût des énergies renouvelables diminue rapidement et que de nombreux pays visent à protéger au moins $30 \%$ du sol et des océans du monde d'ici 2030 (11).

Mais ces promesses sont des vœux pieux. Les cibles sont faciles à établir, mais difficiles à respecter. Elles doivent être assorties de plans crédibles à court et à long terme pour accélérer l'application de technologies plus propres et transformer les sociétés. Les plans de réduction des émissions de gaz à effet de serre ne tiennent pas adéquatement compte des enjeux de santé (12). On craint de plus en plus qu'une hausse de la température mondiale de plus de $1,5^{\circ} \mathrm{C}$ soit malheureusement perçue comme inévitable, voire acceptable, par certains membres puissants de la communauté mondiale (13). À cet égard, les stratégies actuelles visant à ramener les émissions à zéro d'ici le milieu du siècle misent de façon irréaliste sur une hypothétique acquisition de capacités hors du commun pour éliminer les gaz à effet de serre de l'atmosphère $(14,15)$.
Cette mollesse signifie que les hausses de la température augmenteront probablement bien au-delà de $2{ }^{\circ} \mathrm{C}(16)$, une catastrophe pour la santé et l'équilibre de la planète. II est en outre déplorable que la destruction des milieux naturels ne reçoive pas autant d'attention que la dimension strictement climatique de la crise : toutes les cibles mondiales pour rétablir la biodiversité avant 2020 ont été ratées (17). II s'agit d'une crise environnementale globale (18).

Les professionnels de la santé s'unissent aux scientifiques de l'environnement, aux entreprises et à beaucoup d'autres et rejettent l'hypothèse que ce scénario est inévitable. II faut en faire plus maintenant, à Glasgow et à Kunming, et dans les prochaines années. Nous nous joignons aux professionnels de la santé de partout sur la planète qui ont déjà répondu à l'appel à l'action pour la mise en place rapide des mesures nécessaires (19).

L'équité doit être au centre de la réponse globale. Contribuer au partage équitable de l'effort mondial signifie que les engagements vis-à-vis des réductions doivent tenir compte de la contribution cumulative et historique de chaque pays aux émissions, de même que de ses émissions courantes et de sa capacité d'y répondre. Les pays riches devront réduire plus rapidement leurs émissions, et aller au-delà des cibles proposées d'ici $2030(20,21)$ pour atteindre la carboneutralité avant 2050. Des cibles similaires et des mesures d'urgence sont requises pour prévenir la perte de biodiversité et la destruction à grande échelle du monde naturel.

Pour atteindre ces cibles, les gouvernements doivent apporter des changements fondamentaux à l'organisation de nos sociétés et de nos économies et nous devons modifier notre mode de vie. La stratégie actuelle qui consiste à encourager les marchés à adopter des technologies plus propres ne suffit pas. Les gouvernements doivent intervenir pour appuyer une refonte des systèmes de transport, des villes, de la production et de la distribution alimentaires, des marchés financiers, des systèmes de santé et beaucoup plus. Une coordination mondiale est requise pour que cette course aux technologies plus propres ne se fasse pas au détriment de l'environnement et au prix d'une plus grande exploitation humaine.

Plusieurs gouvernements ont répondu à la menace de la pandémie de COVID-19 par un financement sans précédent. La crise environnementale demande une réponse d'urgence similaire : il faudra des investissements bien supérieurs à ceux qui sont envisagés ou appliqués actuellement dans le monde. Mais ces investissements rapporteront énormément aux plans sanitaires et économiques, notamment : emplois de qualité, réduction de la pollution atmosphérique, augmentation de l'activité physique et meilleur accès au logement et à la 
nourriture. À elle seule, l'amélioration de la qualité de l'air entraînerait assez de bienfaits sur la santé pour contrebalancer les coûts globaux de la réduction des émissions (22).

Ces mesures amélioreront aussi les déterminants sociaux et économiques de la santé, qui ont pu rendre certaines populations plus vulnérables à la pandémie de COVID-19 (23). Ces changements devront toutefois se faire sans un retour à des politiques d'austérité délétères et en combattant les inégalités économiques et politiques à l'intérieur des pays et entre eux.

\section{La coopération se fonde sur un engagement plus ferme de la part des pays riches}

Les pays qui ont le plus contribué à la crise environnementale ont le devoir d'en faire plus pour aider les pays à revenus faibles et moyens à devenir des sociétés plus propres, plus saines et plus résilientes. Les pays riches doivent respecter et dépasser leur remarquable engagement à verser 100 milliards de dollars par année pour compenser les lacunes de 2020, et accroître leurs contributions jusqu'à 2025 et au-delà. Le financement doit être destiné également à des mesures d'atténuation et d'adaptation, et notamment à l'amélioration de la résilience des systèmes de santé.

Le financement devrait se faire sous forme de subventions plutôt que de prêts, et passer par le développement des capacités locales et une réelle autonomisation des communautés, en parallèle avec une remise des dettes importantes qui paralysent de si nombreux pays à revenus faibles. II faut mobiliser davantage de fonds pour compenser les pertes et les torts inévitables causés par la crise environnementale.

En tant que professionnels de la santé, nous devons faire le maximum pour assurer une transition vers un monde durable, plus juste, résilient et en meilleure santé, tout en luttant contre les effets négatifs de la crise environnementale. Nous devons contribuer de manière proactive à empêcher que la situation mondiale ne s'aggrave et agir sur les racines de cette crise. II faut tenir les leaders mondiaux responsables et continuer de faire de l'éducation au sujet des conséquences de la crise sur la santé. Nous devons unir nos efforts pour créer des systèmes de santé viables au plan environnemental avant 2040, en reconnaissant que cela modifiera la pratique clinique. Les établissements de santé se sont déjà dessaisis de plus de 42 milliards de dollars d'actifs dans les carburants fossiles; d'autres devraient leur emboîter le pas (4).

La plus grande menace à la santé publique mondiale est l'inertie des leaders mondiaux face à la menace d'une hausse de la température globale de plus de $1,5^{\circ} \mathrm{C}$ et à la détérioration des écosystèmes. Des changements urgents s'imposent à l'échelle des sociétés pour que le monde soit plus juste et en meilleure santé. En tant que rédacteurs en chef de revues médicales, nous enjoignons les gouvernements et autres responsables d'agir, et de faire de 2021 l'année où la trajectoire du monde s'est finalement redressée.

\section{Intérêts concurrents}

Les auteurs ont lu et compris la politique du BMJ sur la déclaration des intérêts. Fiona Godlee fait partie du comité exécutif de la UK Health Alliance on Climate Change et elle est fiduciaire de l'Eden Project. Richard Smith est président de Patients Know Best, et détient des actions de UnitedHealth Group; il a été consultant pour Oxford Pharmagenesis et il est président de la Lancet Commission on the Value of Death. Aucun autre intérêt n'a été déclaré.

\section{Provenance et révision par les pairs}

Sollicité; sans révision externe par des pairs.

\section{Note}

Cet éditorial est publié dans plusieurs revues internationales.

Voir la liste complète ici : https://www.bmj.com/content/ full-list-authors-and-signatories-climate-emergency-editorialseptember-2021

Il s'agit d'un article en libre accès distribué conformément aux modalités de la licence Creative Commons Attribution (CC BY-NC-ND 4.0), qui en permet la distribution, la modification, l'adaptation et l'exploitation à des fins commerciales, à la condition que la publication originale soit adéquatement citée. Voir : https://creativecommons.org/licenses/by-nc-nd/4.0/deed. fr.

\section{Références}

1. World Medical Association, International Council of Nurses, ISDE International, et al. In support of a \#HealthyRecovery. May 26, 2020. https://healthyrecovery.net (accédé août 17, 2021).

2. Intergovernmental Panel on Climate Change. Summary for policymakers. Global warming of $1.5^{\circ} \mathrm{C}$. An IPCC special report on the impacts of global warming of $1.5^{\circ} \mathrm{C}$ above pre-industrial levels and related global greenhouse gas emission pathways, in the context of strengthening the global response to the threat of climate change, sustainable development, and efforts to eradicate poverty. 2018. https://www.ipcc.ch/sr15/ (accédé août 17, 2021).

3. Intergovernmental Science-Policy Platform on Biodiversity and Ecosystem Services. Summary for policymakers: the global assessment report on biodiversity and ecosystem services. 2019. https://ipbes.net/sites/default/files/2020-02/ ipbes_global_assessment_report_summary_for_ policymakers_en.pdf (accédé août 17, 2021).

4. Watts N, Amann M, Arnell N, et al. The 2020 report of the Lancet Countdown on health and climate change: responding to converging crises. Lancet 2021; 397: 129-70. 
5. Rocque RJ, Beaudoin C, Ndjaboue R, et al. Health effects of climate change: an overview of systematic reviews. BMJ Open 2021; 11: e046333.

6. Haines A, Ebi K. The imperative for climate action to protect health. N Engl J Med 2019; 380: 263-73.

7. UN Environment Programme, International Livestock Research Institute. Preventing the next pandemic: zoonotic diseases and how to break the chain of transmission. 2020. https://72d37324-5089-459c-8f70-271d19427cf2.filesusr. com/ugd/056cf4_b5b2fc067f094dd3b2250cda15c47acd.pdf (accédé août 17, 2021).

8. Intergovernmental Panel on Climate Change. Summary for policymakers. Climate change and land: an IPCC special report on climate change, desertification, land degradation, sustainable land management, food security, and greenhouse gas fluxes in terrestrial ecosystems. 2019. https://www.ipcc.ch/srccl/chapter/summary-forpolicymakers/ (accédé août 17, 2021).

9. Lenton TM, Rockström J, Gaffney O, et al. Climate tipping points—too risky to bet against. Nature 2019; 575: 592-95.

10. Wunderling N, Donges JF, Kurths J, Winkelmann R. Interacting tipping elements increase risk of climate domino effects under global warming. Earth Syst Dynam 2021; 12: 601-19.

11. High Ambition Coalition. 2021. https://www. hacfornatureandpeople.org (accédé août 17, 2021).

12. Global Climate and Health Alliance. Are national climate commitments enough to protect our health? https:// climateandhealthalliance.org/initiatives/healthy-ndcs/ndcscorecards/ (accédé août 17, 2021).

13. Thunberg G, Neubauer L, Charlier A, et al. Climate strikers: open letter to EU leaders on why their new climate law is "surrender". Carbon Brief, March 3, 2020. https://www. carbonbrief.org/climate-strikers-open-letter-to-eu-leaderson-why-their-new-climate-law-is-surrender (accédé août 17, 2021).
14. Fajardy M, Köberle A, MacDowell N, Fantuzzi A. BECCS deployment: a reality check. Grantham Institute briefing paper 28. January, 2019. https://www.imperial.ac.uk/media/ imperial-college/grantham-institute/public/publications/ briefing-papers/BECCS-deployment---a-reality-check.pdf (accédé août 17, 2021).

15. Anderson K, Peters $G$. The trouble with negative emissions. Science 2016; 354: 182-83.

16. Climate action tracker. 2021. https://climateactiontracker. org (accédé août 17, 2021).

17. Secretariat of the Convention on Biological Diversity. Global biodiversity outlook 5. 2020. https://www.cbd.int/gbo5 (accédé août 17, 2021).

18. Steffen W, Richardson K, Rockström J, et al. Planetary boundaries: guiding human development on a changing planet. Science 2015; 347: 1259855.

19. UK Health Alliance. Our calls for action. 2021. http://www. ukhealthalliance.org/cop26/ (accédé août 17, 2021).

20. Climate Action Tracker. Warming projections global update: May 2021. https://climateactiontracker.org/documents/853/ CAT_2021-05-04_Briefing_Global-Update_Climate-SummitMomentum.pdf (accédé août 17, 2021).

21. UN Environment Programme. Emissions gap report 2020. Nairobi: United Nations Environment Programme, 2020.

22. Markandya A, Sampedro J, Smith SJ, et al. Health co-benefits from air pollution and mitigation costs of the Paris Agreement: a modelling study. Lancet Planet Health 2018; 2: e126-33.

23. Paremoer L, Nandi S, Serag H, Baum F. Covid-19 pandemic and the social determinants of health. BMJ 2021; 372: n129. 